\title{
Blogs médicos como fuente de formación e información. El ejemplo del blog Pediatría basada en pruebas
}

\author{
J. González de Dios ${ }^{a}$, M. González Muñoz , P. González Rodríguez, \\ M. J. Esparza Olcina ${ }^{d}$, J. C. Buñuel Álvarez ${ }^{e}$
}

Publicado en Internet: 21-marzo-2013

Javier González de Dios javier.gonzalezdedios@gmail.com

Palabras clave:

- Blog

- Formación

- Información

- Internet

- Pediatría

- Red social
Los blogs se han convertido en una potencial fuente de información y formación en Medicina, en general, y en Pediatría, en particular, máxime en el actual entorno sanitario de la "web social". La experiencia personal de los autores en relación con el blog Pediatría basada en pruebas ha permitido analizar el interés temático de los lectores del blog, así como los flujos de comunicación en el entorno de la "blogosfera" pediátrica española.

Se analizan las 1311 entradas publicadas en el blog Pediatría basada en pruebas durante el periodo de revisión (de mayo de 2008 a septiembre de 2012), con un estudio pormenorizado de las 105 entradas que han recibido más de 1000 visitas.

Los temas que más interesan a los lectores del blog Pediatría basada en pruebas se concentran en seis grupos: 1) enfermedades infecciosas (gripe, meningitis, sepsis, sarampión, varicela, vacunas, etc.); 2) Perineonatología (temas de la gestación y del recién nacido, algunos volcados en dos secciones ya consolidadas como Propositus y Neo-Dividencias); 3) temas sociales (tabaquismo, accidentes, inmigración, acoso escolar, etc.); 4) guías e informes de sociedades científicas; 5) secciones ya establecidas del blog (con cinco consolidadas: Propositus, Neo-Dividencias, Formación e Información en Pediatría, Resumen de la blogosfera pediátrica y Cine y Pediatría), y 6) polémicas (temas de especial debate social y/o médico y que corresponden con los más comentados, y donde se incluyen temas como el aborto, la circuncisión neonatal, grupos antivacunas, mala praxis sanitaria, etc.).

Teniendo en cuenta que el entorno de los blogs ya es considerado por la propia Asociación Española de Pediatría como una fuente de información fiable, nuestro compromiso en el blog es construir esta información de la mejor forma posible. Y la mejor forma posible inicial es conocer la dinámica de nuestros lectores y, con ello, y sin dejar de ser rigurosos y éticos, podemos (y debemos) plantear como válida esta forma de transmitir formación e información a nuestros colegas de profesión, a pacientes y familias, y a la sociedad en general.

\section{Medical blogs as a source of training and information: the example of the blog "Pediatría basada en pruebas"}

Blogs have become a potential source of information and training in medicine in general and in pediatrics in particular, especially in the present health care environment in the social web. The authors' personal experience relating to the blog Pediatría basada en pruebas has made possible to analyze the readers' interests, as well as the communication movements around the Spanish pediatric blogosphere.

The 1311 public entries from May 2008 to September 2012 are analyzed, with a detailed study of the 105 posts with more than 1000 visits.

The subjects that interest the most to the readers of Pediatría basada en pruebas blog gather around six groups: 1) infectious diseases (flu, meningitis, septicemia, measles, chickenpox, vaccinations, etc.); 2) peri-neonatology (pregnancy and newborn subjects, some of them issued in two established sections named Propositus and Neo-Dividencias; 3 ) social subjects (smoking, injuries, immigration, bulling, etc.); 4) guidelines and reports of scientific societies; 5) established sections in the blog (five of them consolidated: Propositus, Neo-Dividencias, Training and Information in Pediatrics, Pediatric blogosphere summary and Cinema and Pediatrics; 6) controversies (subjects of special social and/or healthcare debate, that are the more commented ones and include subjects such as abortion, newborn circumcision, anti-vaccination groups, medical malpractice, etc.). 
Key words:

- Blogs

- Training

- Information

- Internet

- Pediatrics

- Social web
Taking into account that blogs are already considered by the Spanish Pediatrics Association itself as a source of information, our compromise with the blog is to build this information in the best possible way. And the initial best possible way is to know our readers' dynamics, and therefore staying rigorous and ethical, we can (and should) consider valid this way of transmitting training and information to our colleagues, patients and families, and to society in general.

\section{INTRODUCCIÓN}

El objetivo principal del quehacer sanitario es ofrecer la mejor atención a la población, a ser posible con un modelo de Medicina cercana, científica y humana. Una Medicina que intente ofrecer la máxima calidad con la mínima cantidad (de intervenciones) y en el lugar más cercano al paciente ${ }^{1}$. Este modelo de Medicina no se puede improvisar, por lo que disponer de unos profesionales adecuadamente formados e informados es un elemento clave para mejorar la calidad asistencial.

Para garantizar la eficiencia del funcionamiento del sistema sanitario, la formación médica debe contemplarse como un continuum desde el pregrado (formación universitaria de seis años), pasando por la formación posgrado (de tres a cinco años de especialización en el modelo de médicos internos y residentes $[M I R])$, que se prolonga durante unos 35-40 años en la etapa conocida como formación médica continuada.

Desde que la revolución informática produjo una explosión del acceso a la información, la "semivida" de los conocimientos biomédicos es aún más corta (debido a la constante renovación de la ciencia, la información se hace obsoleta rápidamente) y la gestión de la información más complicada en un entorno de "infoxicación" (intoxicación por exceso de información). Sin embargo, las nuevas publicaciones no sustituyen a las anteriores, sino que conviven con ellas. Un fenómeno básico de la producción de publicaciones biomédicas es su crecimiento exponencial. Este exceso de información médica imposibilita estar al día en cualquier tema solo con lecturas o suscripciones personales, pues el flujo de ideas en Biomedicina y Ciencias de la Salud se ha hecho internacional, masivo y de gran velocidad de renovación. El médico ha dejado de ser un acumulador de información para convertirse en un buscador de fuentes de información, y cuyo reto es conseguir realizar una buena gestión de su formación sobre el exceso de información médica actual.

Formación e información están muy relacionadas y a ello venimos dedicando una sección concreta en la revista Acta Pediátrica Española, con una orientación especial a la Pediatría (pero con un esquema válido para cualquier especialidad) y que hemos denominado como "Formación e Información en Pediatría”, con múltiples recursos (bases de datos, repositorios y webs) ${ }^{2-4}$. El problema está en transformar adecuadamente la información en conocimiento y el conocimiento en acción para la práctica clínica, en un entorno mayoritario en el que la asistencia ocupa la casi totalidad de la jornada laboral. En la Tabla 1 se exponen algunas claves para la gestión del conocimiento en el siglo XXI dentro de la Biomedicina y Ciencias de la Salud ${ }^{5-7}$. Un aspecto clave es que hoy en día no se puede concebir la información, la formación continuada y la puesta al día sin el recurso de Internet. Muchas son las utilidades de Internet para compartir conocimiento, desde las más conocidas (correo electrónico, listas de distribución, buscadores web, etc.) hasta las más novedosas (como el fenómeno actual de la Web 2.0, con el fenómeno de la "blogosfera" a la cabeza). Pues es justo ahora cuando se está produciendo un nuevo salto en el desarrollo de la red, que pasa de ser un sitio donde almacenar y buscar información, a convertirse en un lugar donde esta información se genera, comparte, modifica y, además, se le agrega valor añadido por parte de los usuarios. Esta nueva forma de utilizar la red se ha venido en llamar Web 2.0 (o "web social") y ya no está llamada a ser una revolución, pues es una realidad. Así pues, la revolución de la 
Tabla 1. Claves para la gestión del conocimiento en el siglo XXI

En Ciencias de la Salud no necesitamos más información, sino más respuestas (menos "ruido documental" y más "música del conocimiento")

La información no es igual a conocimiento (vencer el proceloso "mar de la infoxicación" en busca "del puerto del conocimiento")

La información debe poder estar accesible en distintos tamaños de lectura (el paso de la revista al artículo, y del artículo a las unidades de información de alto valor añadido)

No son precisos más escritores, sino más gestores del conocimiento (investigación orientada a responder las preguntas de clínicos, gestores y pacientes)

La información debe estar en el lugar adecuado y en el momento oportuno (información lista para su uso y en el punto de atención, esto es, "just in time" $y$ "just in case")

De la información estática a la información dinámica (pasar de la Web 1.0 a la Web 2.0, con el camino preparado para la Web 3.0)

información implica que la información deja de ser estática, bajo el concepto Web 1.0 (biblioteca clásica, basada en la autoridad y, principalmente, en forma de texto) a ser dinámica, bajo el concepto Web 2.0 (biblioteca viva, creada por la comunidad de usuarios, en la que los archivos de audio y vídeo empiezan a tener protagonismo, y donde ya apreciamos algunas de sus múltiples caras: redes sociales, weblogs, podcasts, videocasts, webtop, wiki, etc.) $)^{8,9}$. En la Tabla 2 se exponen didácticamente los principales recursos en este entorno de la web social.

Uno de los recursos con progresiva repercusión en la información y comunicación en nuestra sociedad es el blog (diminutivo de weblog), también conocido en español como bitácora (o listado de sucesos). El blog es un sitio web pensado para usarse como una especie de diario online que utilizan uno o varios usuarios para publicar artículos o noticias, los denominados post (entradas). La temática de los blogs es muy variada, desde diarios personales, blogs de opinión, los que recogen lo más interesante de Internet, periodísticos, tecnológicos y médicos, entre otros muchos.

Los blogs suelen actualizarse diariamente y su uso es sencillo. La edición y publicación se realiza directamente en la web y muchos de los servicios, como Wordpress ${ }^{\circledR}$ (http://es.wordpress.com/) o Blogger ${ }^{\circledR}$ (www.blogger.com/) son gratuitos. Los artículos se recopilan cronológicamente apareciendo el más reciente primero. Habitualmente existe una blogroll, una lista de enlaces a otros blogs. Los artículos pueden ser comentados por otros usuarios, facilitando la creación de comunidades en torno a un tema concreto. Además, los contenidos se pueden etiquetar para indexarlos y que su búsqueda sea

Tabla 2. Principales mecanismos de información en la web social

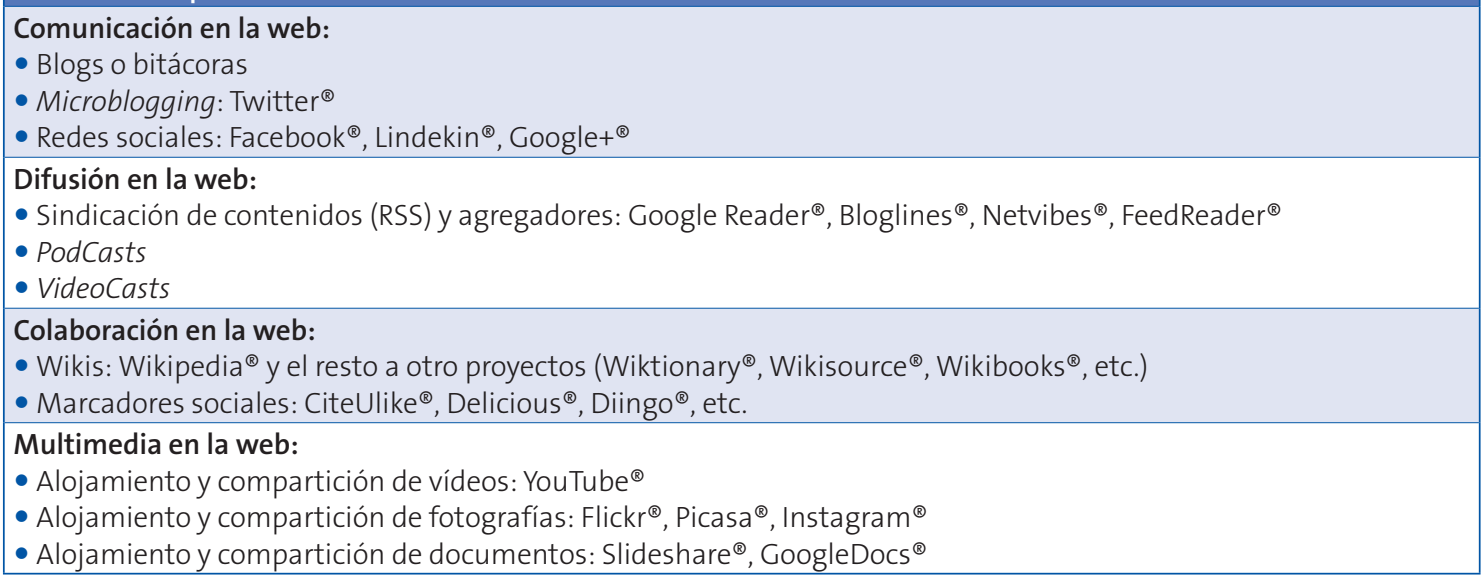


más fácil. Google ${ }^{\circledast}$ ofrece una herramienta para la búsqueda de blogs (www.google.es/blogsearch?hl=es), aunque Technorati ${ }^{\circledR}$ es también un motor de búsqueda de blogs (http://technorati.com/); en Medicina, Medlogs ${ }^{\circledR}$ (www.medlogs.com/) hace esta labor. En este contexto cada vez más patente de introducción de la web social en el entorno sanitario, cabe considerar los blogs como una potencial fuente de formación e información. Y así ha sido considerada por la propia Asociación Española de Pediatría (AEP), que establece enlaces a los principales blogs pediátricos desde su web. La experiencia personal de los autores de este artículo en relación con el blog Pediatría basada en pruebas ha permitido abordar este artículo con dos objetivos principales: 1) desde un punto de vista específico, analizar el interés de los lectores del blog Pediatría basada en pruebas como fuente de información, y 2) desde un punto de vista general, valorar el estado de salud de la blogosfera pediátrica española.

\section{MATERIAL Y MÉTODOS}

- Tipo de estudio: análisis transversal descriptivo del blog Pediatría basada en pruebas (www.pe diatriabasadaenpruebas.com).

- Tiempo de estudio: desde el 09/05/2008, fecha de publicación del primer post (entrada) del blog Pediatría basada en pruebas hasta el 08/09/2012, fecha de la revisión.

- Material de estudio: durante los 52 meses de análisis se habían publicado un total de 1311 entradas en el blog Pediatría basada en pruebas. En los últimos tres años la publicación ha sido regular (con una entrada en cada uno de los 365 días del año), no así en la fase inicial.

- Metodología de revisión: a través del directorio de Blogger ${ }^{\circledR}$ del blog hemos podido revisar todas las entradas y el número de visitas, considerando como criterio de inclusión aquellas entradas que habían sido consultadas por un mínimo de 1000 lectores.

- Variables analizadas en cada entrada: título, fecha de publicación, número de visitas, autor de la entrada, número de comentarios y número de "me gusta" de los lectores.

\section{RESULTADOS}

Sobre el total de 1311 entradas publicadas en el blog Pediatría basada en pruebas durante el periodo de revisión, el 8\% de las mismas (105) ha recibido más de 1000 visitas. En la Tabla 3 se concretan las 25 entradas con mayor número de visitas por parte de los internautas y en la Tabla 4 (disponible solo en www.pap.es) las restantes 80 entradas que han superado las 1000 visitas en la fecha de revisión.

Tal como vemos en la Tabla 3, la única entrada que ha superado las 10000 visitas ha sido la titulada "Cine y Pediatría (73). Pequeña Miss Sunshine y los niños prodigio: ¿estrellas o estrellados?", con 11716 visitas. Le siguen en orden decreciente “ ¿Estamos a salvo del sarampión? Brote epidémico en Granada" (9855 visitas), "Impacto del tabaquismo pasivo en los niños" (9664 visitas), "Actualización del informe sobre gripe pandémica (1 de diciembre de 2009)" (8934 visitas) y "Cine y Pediatría (44). Juno se atreve a debatir sobre el embarazo no deseado en adolescentes" (8920 visitas).

De todas las entradas hasta ahora publicadas, la que ha recibido un mayor número de comentarios posteriores a su publicación ha correspondido a "Nuestros Prematuros, mucho más que estrellas de cine" (17 comentarios y 4185 visitas), "Este blog cumple 2 años" (16 comentarios y 146 visitas) y "Sobre el infanticidio y el aborto selectivo: somos hipócritas" (15 comentarios y 1854 visitas). En la Tabla 5 (disponible solo en www.pap.es) se pueden revisar las 25 entradas que superan los cinco comentarios, ocho de las cuales estaban incluidas entre las más vistas (con más de 1000 visitas), pero el resto no.

De todas las entradas hasta ahora publicadas, la que ha recibido un mayor número de "me gusta" por los seguidores del blog ha correspondido a "Nuestros Prematuros, mucho más que estrellas de cine" (11 "me gusta” y 4185 visitas), "Tosferina, 
Tabla 3. "Top 25" de las entradas más visitadas en el blog Pediatría basada en pruebas

\begin{tabular}{|c|c|c|c|}
\hline $\begin{array}{l}\text { N.o de } \\
\text { visitas }\end{array}$ & $\begin{array}{c}\text { Título de la entrada } \\
\text { Dirección URL }\end{array}$ & $\begin{array}{l}\text { Fecha de } \\
\text { publicación }\end{array}$ & Autor \\
\hline 11716 & $\begin{array}{l}\text { Cine y Pediatría (73). "Pequeña Miss Sunshine" y los niños prodigio: ¿estrellas o } \\
\text { estrellados? } \\
\text { www.pediatriabasadaenpruebas.com/2011/06/cine-y-pediatria-73-pequena- } \\
\text { miss.html }\end{array}$ & $04 / 06 / 2011$ & $J G d D$ \\
\hline 9855 & $\begin{array}{l}\text { ¿Estamos a salvo del sarampión? Brote epidémico en Granada } \\
\text { www.pediatriabasadaenpruebas.com/2010/11/estamos-salvo-del-sarampion- } \\
\text { brote.html }\end{array}$ & $12 / 11 / 2010$ & CBA \\
\hline 9664 & $\begin{array}{l}\text { Impacto del tabaquismo pasivo en los niños } \\
\text { www.pediatriabasadaenpruebas.com/2011/02/impacto-del-tabaquismo-pasivo- } \\
\text { en-los.html }\end{array}$ & 08/02/2011 & CBA \\
\hline 8934 & $\begin{array}{l}\text { Actualización del informe sobre gripe pandémica } \\
\text { www.pediatriabasadaenpruebas.com/2009/12/actualizacion-del-informe-sobre- } \\
\text { gripe.html }\end{array}$ & $01 / 12 / 2009$ & CBA \\
\hline 8920 & $\begin{array}{l}\text { Cine y Pediatría (44). "Juno" se atreve a debatir sobre el embarazo no deseado en } \\
\text { adolescentes } \\
\text { www.pediatriabasadaenpruebas.com/2010/11/cine-y-pediatria-44-juno-se- } \\
\text { atreve.html }\end{array}$ & $13 / 11 / 2010$ & $J G d D$ \\
\hline 6708 & $\begin{array}{l}\text { Guía de Práctica Clínica sobre meningitis bacteriana y sepsis meningocócica } \\
\text { (NICE) } \\
\text { www.pediatriabasadaenpruebas.com/2010/06/guia-de-practica-clinica-sobre. } \\
\text { html }\end{array}$ & $24 / 06 / 2010$ & CBA \\
\hline 6647 & $\begin{array}{l}\text { Antieméticos para la gastroenteritis en niños: eficaces pero... } \\
\text { www.pediatriabasadaenpruebas.com/2009/08/antiemeticos-para-la- } \\
\text { gastroenteritis-en.html }\end{array}$ & $02 / 08 / 2009$ & CBA \\
\hline 5711 & $\begin{array}{l}\text { Guías de Práctica Clínica de la Academia Americana de Pediatría } \\
\text { www.pediatriabasadaenpruebas.com/2009/09/guias-de-practica-clinica-de-la. } \\
\text { html }\end{array}$ & 08/09/2009 & CBA \\
\hline 4245 & $\begin{array}{l}\text { Inhibidores de la bomba de protones, embarazo y teratogenicidad } \\
\text { www.pediatriabasadaenpruebas.com/2011/01/inhibidores-de-la-bomba-de- } \\
\text { protones.html }\end{array}$ & $12 / 01 / 2011$ & $J G d D$ \\
\hline 4185 & $\begin{array}{l}\text { Nuestros Prematuros, mucho más que estrellas de cine } \\
\text { www.pediatriabasadaenpruebas.com/2012/01/nuestros-prematuros-mucho- } \\
\text { mas-que.html }\end{array}$ & 23/01/2012 & $J G d D$ \\
\hline 4148 & $\begin{array}{l}\text { Programa NIDCAP y proyecto Hera: la humanidad y la tecnología pueden y deben } \\
\text { convivir en la atención del prematuro } \\
\text { www.pediatriabasadaenpruebas.com/2009/12/programa-nidcap-y-proyecto- } \\
\text { hera-la.html }\end{array}$ & $31 / 12 / 2009$ & $J G d D$ \\
\hline 3972 & $\begin{array}{l}\text { “Código hipotermia” en el recién nacido con encefalopatía hipóxico-isquémica: } \\
\text { ¿cuándo activarlo en España? } \\
\text { www.pediatriabasadaenpruebas.com/2009/10/codigo-hipotermia-en-el-recien- } \\
\text { nacido.html }\end{array}$ & $31 / 10 / 2009$ & $J G d D$ \\
\hline 3710 & $\begin{array}{l}\text { Educación para la salud sobre asma en centros escolares: un estudio y una } \\
\text { fundación } \\
\text { www.pediatriabasadaenpruebas.com/2011/05/educacion-para-la-salud-sobre- } \\
\text { asma-en.html }\end{array}$ & $12 / 05 / 2011$ & $J G d D$ \\
\hline 3535 & $\begin{array}{l}\text { Estudio y seguimiento racional de los senos dérmicos sacrocoxígeos: cuando } \\
\text { "menos es más" } \\
\text { www.pediatriabasadaenpruebas.com/2011/01/estudio-y-seguimiento-racional- } \\
\text { de-los.html }\end{array}$ & $11 / 01 / 2011$ & $J G d D$ \\
\hline 3488 & $\begin{array}{l}\text { Pre-Guía de Práctica Clínica sobre prevención de accidentes de NICE } \\
\text { www.pediatriabasadaenpruebas.com/2009/11/pre-guia-de-practica-clinica- } \\
\text { sobre.html }\end{array}$ & $12 / 11 / 2009$ & CBA \\
\hline
\end{tabular}




\begin{tabular}{|c|c|c|c|}
\hline $\begin{array}{l}\text { N.o de } \\
\text { visitas }\end{array}$ & $\begin{array}{l}\text { Título de la entrada } \\
\text { Dirección URL }\end{array}$ & $\begin{array}{l}\text { Fecha de } \\
\text { publicación }\end{array}$ & Autor \\
\hline 3291 & $\begin{array}{l}\text { Acoso escolar: todos podemos ayudar } \\
\text { www.pediatriabasadaenpruebas.com/2011/09/acoso-escolar-todos-podemos- } \\
\text { ayudar.html }\end{array}$ & 26/09/2011 & CBA \\
\hline 3110 & $\begin{array}{l}\text { La población infantil inmigrante en España presenta importantes problemas de } \\
\text { salud } \\
\text { www.pediatriabasadaenpruebas.com/2009/11/la-poblacion-infantil-inmigrante- } \\
\text { en.html }\end{array}$ & $19 / 11 / 2009$ & CBA \\
\hline 3108 & $\begin{array}{l}\text { Que no. Que la leche no produce mocos } \\
\text { www.pediatriabasadaenpruebas.com/2011/07/que-no-que-la-leche-no-produce- } \\
\text { mocos.html }\end{array}$ & $21 / 07 / 2011$ & CBA \\
\hline 3036 & $\begin{array}{l}\text { Cine y Pediatría (21). “Mi nombre es Khan”y tengo síndrome de Asperger } \\
\text { www.pediatriabasadaenpruebas.com/2010/06/cine-y-pediatria-21-mi-nombre- } \\
\text { es-khan-y.html }\end{array}$ & 05/06/2010 & $J G d D$ \\
\hline 2954 & $\begin{array}{l}\text { Steve Jobs: in memoriam } \\
\text { www.pediatriabasadaenpruebas.com/2011/10/steve-jobs-in-memoriam.html }\end{array}$ & 06/10/2011 & CBA \\
\hline 2909 & $\begin{array}{l}\text { Cine y Pediatría (97). Películas sobre embarazo en la adolescencia (1) } \\
\text { www.pediatriabasadaenpruebas.com/2011/11/cine-y-pediatria-97-peliculas- } \\
\text { sobre.html }\end{array}$ & $19 / 11 / 2011$ & $J G d D$ \\
\hline 2850 & $\begin{array}{l}\text { Cine y Pediatría (100). } 100 \text { días de cine alrededor de la pediatría, } 100 \text { días de } \\
\text { aprendizaje y de emoción } \\
\text { www.pediatriabasadaenpruebas.com/2011/12/cine-y-pediatria-100-100-dias- } \\
\text { de-cine.html }\end{array}$ & $10 / 12 / 2011$ & $J G d D$ \\
\hline 2631 & $\begin{array}{l}\text { Un año de "Cine y Pediatría" } \\
\text { www.pediatriabasadaenpruebas.com/2011/01/un-ano-de-cine-y-pediatria.html }\end{array}$ & $17 / 01 / 2011$ & JGdD \\
\hline 2615 & $\begin{array}{l}\text { Guías de Práctica Clínica CENETEC (México) } \\
\text { www.pediatriabasadaenpruebas.com/2010/03/guias-de-practica-clinica- } \\
\text { cenetec.html }\end{array}$ & $31 / 03 / 2010$ & CBA \\
\hline 2608 & $\begin{array}{l}\text { Cine y Pediatría (3): George Clooney puso el listón muy alto a “los" pediatras } \\
\text { www.pediatriabasadaenpruebas.com/2010/01/cine-y-pediatria-3-george- } \\
\text { clooney-puso.html }\end{array}$ & $30 / 01 / 2010$ & $J G d D$ \\
\hline
\end{tabular}

CBA: Cristóbal Buñuel Álvarez. JGdD: Javier González de Dios.

movimientos antivacuna y el triunfo de la ignorancia” (10 “me gusta”y 1359 visitas) y "Sobre el infanticidio y el aborto selectivo: somos hipócritas" (10 "me gusta"y 1854 visitas). En la Tabla 6 (disponible solo en www.pap.es) se pueden revisar las 25 entradas que superan los cinco "me gusta", 13 de las cuales estaban incluidas entre las más vistas (con más de 1000 visitas), pero el resto no.

\section{DISCUSIÓN}

Partimos de una realidad constatable (y ya incontestable): tres de cada cuatro usuarios se informan sobre salud en Internet. Se está produciendo la aparición del paciente experto y empoderado, aquel que busca información de salud en la red antes y después de acudir a la consulta del profesional sanitario ${ }^{8,9}$.

Está apareciendo toda una generación que cree que la pantalla es lo real (son los llamados screenagers) y, quizás, no debamos ignorarlos porque "piensen de forma digital". Máxime cuando el 65\% de los jóvenes entre 18 y 24 años (máximos usuarios de Internet) declara que Internet es un medio ideal y preferente para el aprendizaje, la formación y la información. Sobre todo, el auge en el uso de la red ha sido logarítmico en la Web 2.0 o web social, principalmente sus cuatro recursos estreIla: Facebook ${ }^{\circledR}$ Twitter $^{\circledR}$, YouTube $^{\circledR}$ y el mundo de los blogs. 
Se valora en casi 180 millones el número de blogs en el mundo (cifra que queda automáticamente desactualizada cada día que pasa), con el liderazgo de Estados Unidos (casi el 50\% de los blogs) y la Unión Europea (el 30\%). Las principales temáticas son reflexiones personales (18\%) y tecnología y marketing online (14\%); aunque sólo un 2-3\% versa sobre salud, existe un progresivo incremento de esta temática. Se dice que cada día hay 1,5 millones de entradas nuevas en blogs de todo el mundo.

En el campo de la salud, la temática de los blogs es muy variada. En ellos los profesionales crean debates acerca de información de interés, cuentan sus experiencias laborales y exponen sus opiniones, entre otras muchas opciones. Los pacientes también los usan como medio para intercambiar consejos, apoyo, contar sus experiencias, etc. En estos momentos (diciembre de 2012) se contabilizan alrededor de 200 blogs médicos en España, cifra que asciende semana a semana. Una de las áreas de la Medicina más activas en el mundo de los blogs es la Pediatría, conformando lo que se ha venido en llamar como "blogosfera pediátrica". En la Tabla 7 se ofrece un listado de blogs pediátricos editados, bien en español, bien en España. Un mundo, el de los blogs pediátricos, que tiene fuerza y credibilidad, tal como se ha podido confirmar a lo largo de este año 2012 con dos hechos puntuales: la inclusión de los blogs pediátricos como fuente de información en la web de la AEP (si bien con esta nota: "Los siguientes blogs no tienen vinculación directa con la AEP. Su inclusión en esta sección no implica que la AEP comparta necesariamente las opiniones expresadas en ellos") y el premio y accésit del I Premio Merck al mejor blog de salud a dos blogs pediátricos (concretamentea Hij@s de Eva y Adán y a Pediatría basada en pruebas).

Según los resultados de este estudio (al revisar las Tablas 3 y 4 -esta última solo en www.pap.es-), parece que a los lectores del blog Pediatría basada en pruebas (profesionales sanitarios y población general) los temas que más interesan se concentran en seis grupos:

1. Enfermedades infecciosas: entradas relacionadas con gripe, meningitis, sepsis, sarampión, varicela, vacunas, etc.
2. Perineonatología: entradas en relación con temas de la gestación y del recién nacido, algunos volcados en dos secciones ya consolidadas (Propositus y Neo-Dividencias).

3. Temas sociales: entradas relacionadas con tabaquismo, accidentes, inmigración, acoso escolar, etc.

4. Guías e informes de sociedades científicas: entradas que enlazan con guías de práctica clínica de entidades de calidad (AAP, NICE, SIGN, GuíaSalud, CENETEC, etc.), conferencias de consenso, informes de evaluación, etc.

5. Secciones ya establecidas del blog, entre las que se pueden citar al menos cinco: Propositus (sobre las hojas de información para prevención primaria a gestantes y realizadas por el Estudio Colaborativo Español de Malformaciones Congénitas), Neo-Dividencias (sobre las polémicas alrededor de la investigación y la práctica clínica en Perineonatología), Formación e Información en Pediatría (sobre los artículos publicados sobre esta materia en la revista Acta Pediátrica Española), Resumen de la blogosfera pediátrica (sobre lo mejor de la última semana en las entradas que se han publicado en blogs principalmente pediátricos) y Cine y Pediatría (comentarios a películas con valores relacionados con la infancia y adolescencia). Es llamativo el interés alrededor de esta última sección, de forma que Cine y Pediatría abarca 17 de las 105 entradas más visitadas.

6. Polémicas: temas de especial debate social y/o médico y que corresponden con los más comentados (Tabla 5 -solo en www.pap.es-) y donde se incluyen temas como el aborto, la circuncisión neonatal, grupos antivacunas, mala praxis sanitaria, etc.

Aunque el blog tiene un buen número de seguidores (más de 500 en estos momentos) y de lectores, resulta aún poco habitual la interacción, de forma que el mayor número de comentarios (17, según vemos en la Tabla 5 -solo en www.pap.es-) y de "me gusta" (11, según vemos en la Tabla 6-solo en www.pap.es-) a una entrada siguen siendo cifras 
Tabla 7. Listado de blogs pediátricos en español o en España

\begin{tabular}{|l|l|}
\hline Asma, Deporte y Salud & Mi reino por un caballo \\
\hline Bitácora Prevlnfad & Pediatra de Cabecera \\
\hline Casos clínicos de Pediatría & Pediatra Humanista \\
\hline Con Estetoscopio & Pediatras del Centro de Salud Rosa Luxemburgo \\
\hline Diario de una mamá pediatra & Pediatres de Ponent \\
\hline El Blog del Pediatra & Pediatría Basada en Pruebas \\
\hline EL GIPI-Twitter & Pediatría para usuarios \\
\hline El Médico de mi Hij@ & Pediatría Social \\
\hline Grupo de Sueño de la AEPap & Pediatría y Salud \\
\hline Grupo de Vías Respiratorias & Pediátrica \\
\hline Hij@s de Eva y Adán & Perlinfad \\
\hline Las mil y una noches... de Pediatría & Pi Pediatra \\
\hline La otra consulta & Programa de Salud Infantil. Centro de Salud de Olaguibel \\
\hline Maynet & Reflexiones de un pediatra curtido \\
\hline Media Medicina & Sin Estetoscopio \\
\hline Mi pediatra 2.0 & Sociedad Canaria de Pediatría Extrahospitalaria \\
\hline
\end{tabular}

moderadas para lo que podríamos entender como web social. Es reconocido que los blogs más profesionalizados suelen tener menos interacción por parte de los lectores que aquellos que comprenden principalmente a la comunidad de usuarios, pacientes o familiares. Otra reflexión sobre este punto es que no siempre las entradas más leídas son las más comentadas o las que tienen más "me gusta". Todo lo anterior conlleva una meditada reflexión sobres los usos y gustos de lectura de nuestros internautas.

Cabe considerar algunas limitaciones a nuestro estudio, inherentes a los estudios cienciométricos de la red ${ }^{10}:$ no podemos conocer las características de los lectores del blog (profesionales de la salud o pacientes/familiares); los indicadores analizados no son estáticos y es posible que las entradas más actuales mejoren en los indicadores analizados (especialmente las del último trimestre, pues también es cierto que una entrada en un blog tiene una "vida media" muy corta más allá de la primera semana de su publicación).

El blog Pediatría basada en pruebas se ha consolidado como una fuente de información y un recurso muy consultado, como avalan un número de páginas vistas a la semana que oscila entre las 12000 y las 15 000. Por ello, siempre es interesante conocer cuáles son los principales intereses de los lectores (profesionales de la salud, padres y demás usuarios) en su búsqueda de información y formación en temas relacionados con la enfermedad y salud infantojuvenil. Teniendo en cuenta que el entorno de los blogs ya es considerado por la propia AEP como una fuente de información fiable, nuestro compromiso es construir esta información de la mejor forma posible.

El blog Pediatría basada en pruebas, que comenzó hace cuatro años como una afición personal de los autores, se ha ido convirtiendo en un compromiso con nuestros lectores para proporcionar información interesante, veraz y ética. En este sentido, estamos convencidos de que los profesionales sanitarios (y también los hospitales, los centros de salud, las sociedades científicas, etc.) deben dar el salto a la web social... sin miedo y sin pudor. Con contenidos de calidad y estudiando bien lo que ocurre entre emisor (profesionales sanitarios) y receptor (pacientes y familias), para que el mensaje llegue claro y sin distorsiones. Es por ello por lo que, sin dejar de ser rigurosos y éticos, podemos (y debemos) plantear como válida esta forma de transmitir el mensaje a nuestros colegas de profesión, a pacientes y familias, y la sociedad en general.

Con el ejemplo del blog analizado, queremos apoyar una realidad: que los blogs son una nueva 
fuente de formación e información en salud. En esta labor están implicados muchos blogs en Pediatría y en español (Tabla 7). En cualquier caso, siempre conviene hacer de esta realidad un ponderado análisis interno de esta nueva forma de información en Internet (con sus debilidades y fortalezas) y también un análisis externo de su papel en el entorno de la salud (con sus fortalezas y, cómo no, con sus amenazas).

\section{BIBLIOGRAFÍA}

1. Gervas J. La clave para mejorar la clínica: más calidad con el mínimo de cantidad. Gac Med Bilbao. 2006; 103:46-7.

2. González de Dios J, Aleixandre Benavent R. Formación e información en Pediatría: aproximación a la necesidad de los profesionales. Acta Pediatr Esp. 2010;68:235-40.

3. Aleixandre Benavent R, González Alcaide G, González de Dios J, Alonso Arroyo A. Fuentes de información bibliográfica (I). Fundamentos para la realización de búsquedas bibliográficas. Acta Pediatr Esp. 2011 69:131-6

4. González de Dios J, Buñuel Álvarez JC, González Rodríguez P, Aleixandre Benavent R. Fuentes de información bibliográfica (XIV). Sobre "fuentes", "pirámides" y "revoluciones" en la gestión del conocimiento en Pediatría. Acta Pediatr Esp. 2012;70:289-95.

5. Bravo R. La gestión del conocimiento en Medicina: a la búsqueda de la información perdida. An Sist Sanit Nav. 2002;25:255-72.

\section{CONFLICTO DE INTERESES}

Javier González de Dios, José Cristóbal Buñuel Álvarez, Paz González Rodríguez y M. a Jesús Esparza Olcina son los coautores habituales del blog Pediatría basada en pruebas (www. pediatriabasadaenpruebas.com/)

\section{ABREVIATURAS}

AEP: Asociación Española de Pediatría • MIR: médicos internos residentes.

6. González de Dios J, Pérez Sempere A, Aleixandre R. Las publicaciones biomédicas en España a debate (II): las "revoluciones" pendientes y su aplicación a las revistas neurológicas. Rev Neurol. 2007;44:101-12.

7. González de Dios J. El neuropediatra y la medicina basada en la evidencia. Rev Neurol. 2008;47 (Supl 1):75-95

8. Coronado Ferrer S, Peset Mancebo F, Ferrer Sapena A, González de Dios J, Aleixandre Benavent R. Web 2.0 en medicina y pediatría (I). Acta Pediatr Esp. 2011; 69:3-11.

9. Coronado Ferrer S, Peset Mancebo F, Ferrer Sapena A, González de Dios J, Aleixandre Benavent R. Web 2.0 en medicina y pediatría (II). Acta Pediatr Esp. 2011; 69:79-87.

10. González de Dios J, Aleixandre Benavent R. Evaluación de la investigación en Biomedicina y Ciencias de la Salud. Indicadores bibliométricos y cienciométricos. Bol Pediatr. 2007;47:92-110. 